\title{
Model-free leakage index estimation of the blood-brain barrier using dual dynamic susceptibility contrast MRI acquisition
}

\section{Seokha Jin | Hyung Joon Cho}

Department of Biomedical Engineering, Ulsan National Institute of Science and Technology, Ulsan, South Korea

\section{Correspondence}

Hyung Joon Cho, PhD, Department of Biomedical Engineering, Ulsan National Institute of Science and Technology,

50, UNIST-gil, Eonyang-eup, Ulju-gun, Ulsan, South Korea.

Email: hjcho@unist.ac.kr

\section{Funding information}

Korea Health Industry Development Institute, Grant/Award Number: HI14C1135; National Research Foundation of Korea, Grant/Award Numbers: 2018R1A6A1A03025810, 2018M3C7A1056887; 2021 Joint Research Project of Institutes of Science and Technology
Pharmacokinetic $K_{2}$ mapping from dynamic susceptibility contrast (DSC)-MRI can be a sensitive technique for evaluating the vascular permeability of the subtly damaged blood-brain barrier (BBB) in ischemic regions. However, the $K_{2}$ values of ischemic lesions depend upon the selection of the intact $B B B$ reference region. As previous observations suggest that the $\Delta R_{2}{ }^{*}$ curve of pre-loaded DSC-MRI is not significantly affected by the extravasation of contrast agent, dual DSC-MRI acquisitions can be performed to derive the BBB leakage index from the voxel-wise reference input function for ischemic regions. This study aims to demonstrate the robustness of such model-free leakage index estimation in ischemic brains. By configuring the relationship between dual $\Delta R_{2}{ }^{*}$ curves of the intact contralateral brain, the deviation of the measured $\Delta R_{2}{ }^{*}$ curve from the unloaded DSC-MRI with respect to the non-deviated $\Delta R_{2}{ }^{*}$ curve in the pre-loaded DSC-MRI can be quantified as the BBB leakage index. Such model-free leakage index values from rats with transient middle carotid artery occlusion ( $\mathrm{MMCAO})(n=17)$ and normal controls $(n=3)$ were evaluated and compared with conventional $K_{2}$ values with multiple reference regions. Inter-subject leakage index values were also compared with the corresponding $\Delta T_{1}$ map. Evansblue-stained images were used to validate the leakage index. For the TMCAO group, leakage index values correlated well with $\Delta T_{1}$ (Pearson's $r=0.828$ ). The hyperintense area on the leakage index map matched well with the corresponding Evans-blue-stained area (Dice correlation $=0.626$ ). The slopes of the scatter-plot from the leakage index (0.97-1.00) were observed to be more robust against changes in the reference region than those from conventional $K_{2}$ values (0.94-1.07). In a subtly damaged BBB $\mathrm{tMCAO}$ model, model-free evaluation of vascular permeability using dual DSC-MRIs would provide a consistent measure of inter-subject vascular permeability.

KEYWORDS

DSC-MRI, DCE-MRI, Vascular permeability, Ischemic stroke, Blood Brain Barrier 


\section{1 | INTRODUCTION}

In ischemic stroke, the disruption of the blood-brain barrier (BBB) is known to significantly contribute to subsequent neurological impairments. ${ }^{1-3}$ BBB changes in ischemia/reperfusion are dynamic and complex, and thus it is expected that dynamical and quantitative monitoring of BBB integrity could potentially lead to safer and improved outcomes with therapeutic measures, such as thrombolysis. ${ }^{4,5}$ Cerebral MRI for vascular permeability measurement is an essential imaging biomarker to evaluate the integrity of the BBB. ${ }^{6-8}$ For example, dynamic contrast-enhanced (DCE)-MRI measures the signal increase from Gd-based MR contrast agent (CA) injection and estimates the pharmacokinetic model-based transfer constant $\left(K^{\text {trans }}\right)$. Although this $K^{\text {trans }}$ reflects the mixture of vascular permeability and flow, for a low permeability condition like the early stage of ischemic stroke the $K^{\text {trans }}$ may mainly represent vessel permeability. ${ }^{9,10}$ DCE-MRI relies upon $T_{1}$ relaxation time shortening due to leakage of the CA into interstitial spaces. As it requires a certain amount of CA accumulation for $T_{1}$ values to change, its sensitivity may be limited in detecting subtle BBB damage, as in the early stage of ischemic stroke, within short DCE-MRI acquisition time (<10 min). ${ }^{11}$

Dynamic susceptibility contrast (DSC)-MRI is a $T_{2}{ }^{*}$-based or $T_{2}$-based technique that is typically used for evaluating cerebral blood volume, cerebral blood flow (CBF), and mean transit time in capillaries. ${ }^{12}$ DSC-MRI has a basic assumption that the CA is not extravasated from blood vessels, ${ }^{13-15}$ but numerous studies have used the $K_{1}, K_{2}$ model to study the effects of CA extravasation on the DSC-MRI signal in brains with BBB damage, especially for tumors and strokes. ${ }^{8,16,17}$ Specifically, to derive corresponding $K_{1}$ and $K_{2}$ values of lesions with a BBB disruption, the $\Delta R_{2}{ }^{*}$ curve of the intact contralateral BBB region should be pre-determined as a reference input function. However, the averaged $\Delta R_{2}{ }^{*}$ curve of the intact BBB reference region may not represent heterogeneous $\Delta R_{2}{ }^{*}$ curves in the contralateral (normal) brain, which has various $\Delta R_{2}{ }^{*}$ curve patterns depending on local anatomy. Therefore, the $K_{2}$ values provide model-dependent relative values depending on the selection of the intact BBB "reference region." To compensate for such problems, the shape of the $\Delta R_{2}{ }^{*}$ curve from the reference region is often adjusted by an additional time scaling factor and time offset. ${ }^{17}$ However, the introduction of a time scaling factor and offset cannot fully describe the shape of the $\Delta R_{2}{ }^{*}$ curve from the reference region, and $K_{2}$ is still a model-derived value depending on the selection of the reference region. Therefore, if the voxel-wise reference input function, ie the non-deviated $\Delta R_{2}{ }^{*}$ curve for each voxel of the ischemic lesion with BBB leakage, can be estimated, the dependence of DSCMRI variable values on the choice of the reference region will be minimized to potentially provide a model-free BBB leakage index.

To directly measure the non-deviated $\Delta R_{2}{ }^{*}$ curve of the regions with a disrupted BBB, pre-loaded DSC-MRI acquisition is a potential candidate method. ${ }^{18-21}$ As the extravasating CAs would cause $T_{1}$ reduction in tissue and consequently positively enhance the DSC-MRI signal, several previous studies have shown that extravasated CAs from the unloaded DSC-MRI (the first DSC-MRI) prevent such positive signal enhancement due to $T_{1}$ reduction in lesions with a damaged BBB during the pre-loaded DSC-MRI (the second DSC-MRI). No significant cerebral blood volume differences in the blood pool CA when using DSC-MRI and pre-loaded DSC-MRI were subsequently observed. ${ }^{18,19}$ Such observations suggest that the $\Delta R_{2}{ }^{*}$ curve of pre-loaded DSC-MRI is not significantly affected by the extravasation of the CA. Therefore, by performing two sequential DSC-MRIs, the $\Delta R_{2}{ }^{*}$ curve of the pre-loaded DSC-MRI could be considered a voxel-wise reference input function for the $\Delta R_{2}{ }^{*}$ curve of the unloaded DSC-MRI in ischemic regions. By configuring the relationship between the unloaded and pre-loaded $\Delta R_{2}{ }^{*}$ curves of the intact contralateral brain, the deviation of the measured $\Delta R_{2}{ }^{*}$ curve from the unloaded DSC-MRI with respect to the non-deviated $\Delta R_{2}{ }^{*}$ curve from the pre-loaded DSC-MRI can then be quantified as a BBB leakage index.

In this study, we propose a method to estimate vascular permeability that is robust to the reference region without pharmacokinetic modeling by using sequentially implemented dual DSC-MRI acquisitions. To verify the proposed method, preclinical rodent studies were performed step by step. First, the feasibility of the proposed method was confirmed in normal control rats. Second, to demonstrate the robustness of the leakage index against the choice of reference areas, the $K_{2}$ and leakage index based on multiple reference regions were directly compared in $1 \mathrm{~h} M C A O$ with $1 \mathrm{~d}, 3 \mathrm{~d}$, or $5 \mathrm{~d}$ reperfusion rats. Finally, to address the accuracy in estimating the extension and the level of BBB leakage of the proposed method in ischemic lesions, the regions with disrupted BBB on the leakage index map were mutually validated with corresponding histological Evans blue staining and $\Delta T_{1}$ maps, and inter-subject variations of leakage index values were compared with corresponding $\Delta T_{1}$ values.

\section{2 | MATERIALS AND METHODS}

\section{1 | Animal preparation}

All experiments were approved by the institutional animal care and use committee. For the animal experiment, 7-week-old female SpragueDawley (SD) rats from Orient Bio (Gyeonggi, Republic of Korea) were used. The experimental group $(n=17)$ and control group ( $n=3)$ consisted of ischemic stroke surgery rats and normal rats, respectively. The transient middle carotid artery occlusion (tMCAO) group underwent $1 \mathrm{~h}$ MCAO surgery with intraluminal monofilament $(0.35 \mathrm{~mm}$ diameter filament, Doccol, Sharon, MA) with $1 \mathrm{~d}(n=6), 3 \mathrm{~d}(n=6)$, and $5 \mathrm{~d}(n=5)$ of reperfusion depending on their group. During the $7 \mathrm{~T} \mathrm{MRI} \mathrm{(Bruker} \mathrm{BioSpin,} \mathrm{Ettlingen,} \mathrm{Germany)} \mathrm{scan,} \mathrm{rats} \mathrm{were} \mathrm{anesthetized} \mathrm{with} \mathrm{1-2 \%} \mathrm{isoflurane.}$ The body temperature of rats was maintained at $37^{\circ} \mathrm{C}$ by circulating warm water. Sequential dual DSC-MRI was performed to evaluate vascular permeability with a same injection dose and pulse sequence. Besides, to confirm the edematous area and region with damaged BBB, the apparent 
diffusion coefficient (ADC) and longitudinal $T_{1}$ difference $\left(\Delta T_{1}\right)$ maps were acquired. The $\Delta T_{1}$ maps were calculated by subtracting two different $T_{1}$ maps. After MRI scanning, a rat in the $3 \mathrm{~d}$ reperfusion group was used for Evans blue staining. Also, for DCE-MRI, $0.3 \mathrm{mmol} / \mathrm{kg}$ of Gd-DOTA was injected into one rat in the $1 \mathrm{~d}$ reperfusion model.

\section{2 | MRI protocols}

$T_{2}$-weighted MR images were obtained using rapid acquisition with relaxation enhancement (RARE) with the following parameters: repetition time $\left(T_{R}\right)=5000 \mathrm{~ms}$, RARE factor $=4$, effective echo time $\left(T_{\mathrm{E}}\right)=30 \mathrm{~ms}$, number of averages $(\mathrm{NA})=2$, field of view $(\mathrm{FOV})=30 \times 30 \mathrm{~mm}^{2}, \mathrm{matrix}$ size $=256 \times 256$, number of slices (NS) $=3$, slice gap (SG) $=0.2 \mathrm{~mm}$, and slice thickness $(\mathrm{ST})=1 \mathrm{~mm}$.

ADC maps were acquired using diffusion-weighted echo planar imaging (EPI) with the following parameters: $T_{\mathrm{R}}=5000 \mathrm{~ms}$; number of segments $=4$; effective $T_{\mathrm{E}}=20 \mathrm{~ms} ; b$ values $=200,400,600$, and $1000 \mathrm{~s} / \mathrm{mm}^{2} ; \mathrm{NA}=1 ; \mathrm{FOV}=30 \times 30 \mathrm{~mm}^{2} ; \mathrm{matrix}$ size $=96 \times 96 ; \mathrm{NS}=3$; $\mathrm{SG}=0.2 \mathrm{~mm}$; and ST $=1 \mathrm{~mm}$. Three ADC maps in the $x, y$, and $z$ planes were averaged to obtain the mean ADC values.

$T_{1}$ maps were obtained using RARE with variable $T_{R}$ (RAREVTR) with the following parameters: $T_{R}=80,150,200,400,800,1200,1600$, 2000, 2500, 3000, and $4500 \mathrm{~ms} ; \mathrm{RARE}$ factor $=4$; effective $T_{E}=5.9 \mathrm{~ms} ; \mathrm{NA}=1 ; \mathrm{FOV}=30 \times 30 \mathrm{~mm}^{2} ;$ matrix size $=96 \times 96 ; \mathrm{NS}=3$; $\mathrm{SG}=0.2 \mathrm{~mm}$; and ST $=1 \mathrm{~mm}$. The total acquisition time of RAREVTR was $10 \mathrm{~min}$. $T_{1}$ maps were acquired before and 5, 15, and $30 \mathrm{~min}$ after flushing of the injection line with saline. The flushing was performed by injecting saline as much as the dead volume of injection line ( $90 \mu \mathrm{L}$ ) after unloaded DSC-MRI.

DSC-MRI perfusion maps were acquired using a gradient-echo EPI sequence with shortened repetition time to capture the $T_{1}$ effect of extravasating CA. The detailed pulse sequence parameters are as follows: $T_{\mathrm{R}}=300 \mathrm{~ms}$, effective $T_{E}=17 \mathrm{~ms}, \mathrm{NA}=1, \mathrm{FOV}=30 \times 30 \mathrm{~mm}{ }^{2}$, matrix size $=96 \times 96, \mathrm{NS}=3, \mathrm{SG}=0.2 \mathrm{~mm}, \mathrm{ST}=1 \mathrm{~mm}$, bandwidth $=3.5 \times 10^{5} \mathrm{~Hz}$, number of segments $=1$, flip angle (FA) $=30^{\circ}$, number of repetitions $(N R)=800$, and temporal resolution $=0.3 \mathrm{~s}$. Baseline and total acquisition times for DSC-MRI were 1 min (200 images) and 4 min, respectively. For the dual DSC-MRIs, two DSC-MRI experiments were performed sequentially. For the unloaded DSC-MRI (the first DSC-MRI), $0.2 \mathrm{mmol} / \mathrm{kg}$ of Gd-DOTA was injected. After the first injection, the remaining Gd-DOTA ( $0.1 \mathrm{mmol} / \mathrm{kg})$ in the dead volume $(90 \mu \mathrm{L})$ of the injection line was flushed with $90 \mu \mathrm{L}$ of saline. The pre-loaded DSC-MRI (the second DSC-MRI) was performed 40 min (with three $T_{1}$ measurements between dual DSC-MRIs) after the flushing of the injection line. The injection dose and pulse parameters of the pre-loaded DSC-MRI were identical to the unloaded DSC-MRI.

DCE-MRI data were acquired using fast low-angle shot (FLASH) with the following parameters: $T_{R}=35 \mathrm{~ms}, T_{E}=1.9 \mathrm{~ms}, \mathrm{NA}=1$, $\mathrm{FOV}=30 \times 30 \mathrm{~mm}^{2}$, matrix size $=96 \times 96, \mathrm{NS}=3, \mathrm{SG}=0.2 \mathrm{~mm}, \mathrm{ST}=1 \mathrm{~mm}, \mathrm{FA}=30^{\circ}, \mathrm{NR}=180$, and temporal resolution $=3.36 \mathrm{~s}$. The dose of Gd-DOTA was $0.3 \mathrm{mmol} / \mathrm{kg}$. Baseline and total acquisition times for DCE-MRI were $1 \mathrm{~min}$ (18 images) and 9 min, respectively. DCE-MRI was performed with one rat from the $1 \mathrm{~d}$ reperfusion group without additional DSC-MRI experiment.

\subsection{Ex vivo assessment of BBB breakdown and extent of ischemia}

To confirm the region of BBB disruption, one rat from the $3 \mathrm{~d}$ reperfusion group was stained with Evans blue. ${ }^{22}$ While the rat was anesthetized, $2 \mathrm{~mL} / \mathrm{kg}$ of Evans blue solution (4\%) was injected via the tail vein. The brains of rats were perfused with saline 30 min after the injection and fixed with 4\% PFA for $2 \mathrm{~d}$. The brains were embedded in OCT compound and axially sliced in $100 \mu \mathrm{m}$ thick sections using a cryostat (Leica). After the sections were mounted and coverslipped, fluorescence images were acquired using a ZEISS Axio Zoom.V16 microscope with 63 ZEISS filter (excitation/emission wavelength $=572 / 629 \mathrm{~nm}$ ). The spatial resolution of the acquired image was $1 \mu \mathrm{m} \times 1 \mu \mathrm{m}$.

\subsection{Data analysis}

To confirm the edematous area, the ADC map in each direction $(x, y, z)$ was calculated using a mono-exponential function. ${ }^{23}$ The three $A D C$ maps were averaged to obtain the mean ADC values.

To evaluate vascular permeability, $K_{2}$ and $K^{\text {trans }}$ were computed from DSC-MRI and DCE-MRI, respectively. The DSC-MRI signal was converted to the $\Delta R_{2}{ }^{*}$ curve $\left(=-1 / T_{E} \ln \left[S(t) / S_{\text {pre] }}\right.\right.$, where $S(t)$ is the DSC-MRI signal and $S_{\text {pre }}$ is an averaged signal from the pre-injection of DSC-MRI. To obtain $K_{2}$ values, each voxel of the $\Delta R_{2}{ }^{*}$ curve was fitted using the following equation ${ }^{17}$ :

$$
\Delta R_{2}^{*}(t)=K_{1} \overline{\Delta R_{2}^{*}\left(\frac{t+\tau}{\alpha}\right)}-K_{2} \int_{0}^{t} \overline{\Delta R_{2}^{*}\left(\frac{t^{\prime}+\tau}{\alpha}\right) d t^{\prime}}
$$


(A) Reference (intact) regions on the unloaded and preloaded DSC-MRIs

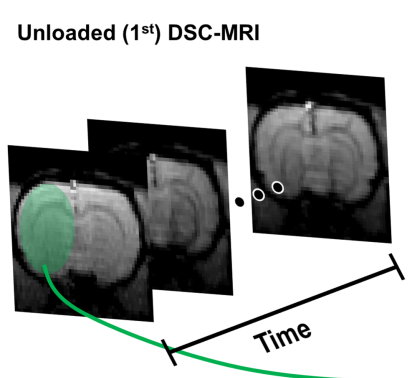

(B) Calculation of a conversion matrix between reference regions
(D) Comparison between measured and estimated unloaded DSC-MRI curves for an intact voxel

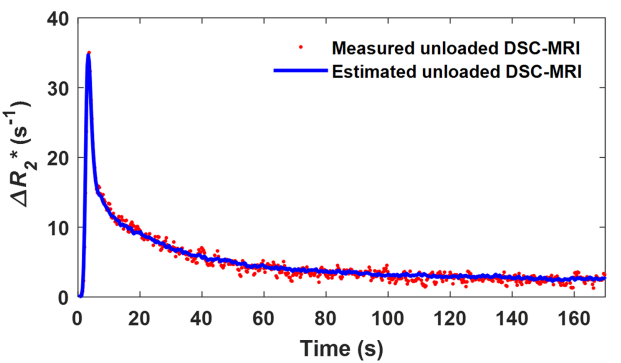
$\mathrm{n}$ voxels

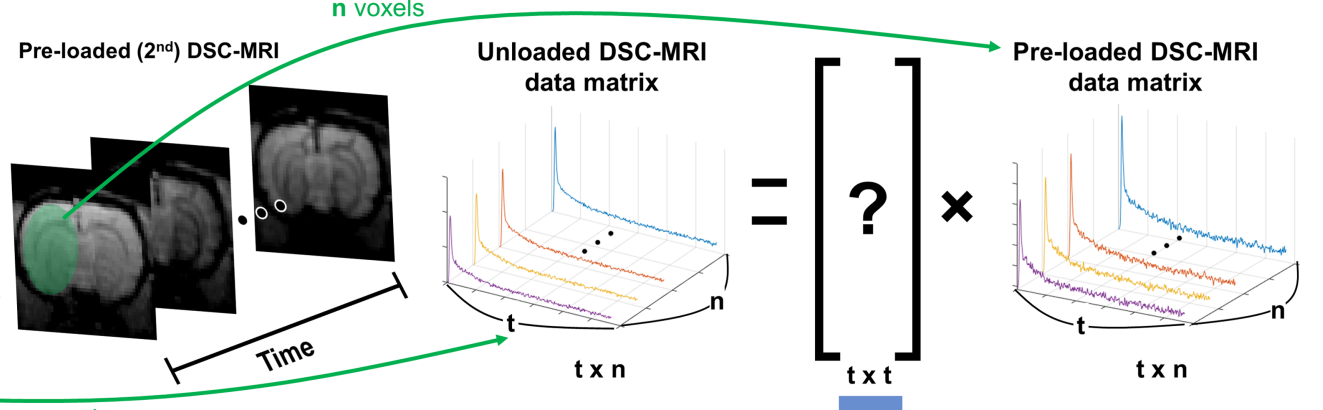

$\mathrm{n}$ voxels

(C) Estimation of unloaded DSC-MRI curve from pre-loaded DSC-MRI

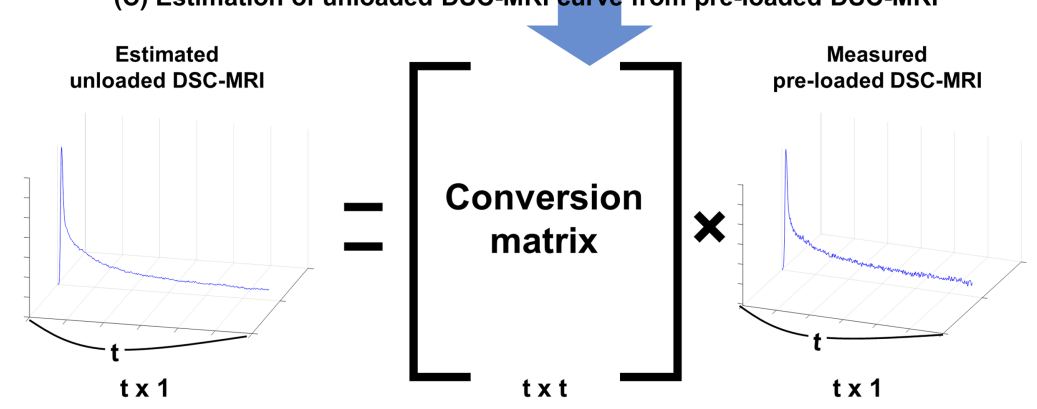

FIGURE 1 The processes of estimating the non-deviated unloaded DSC-MRI curve from the pre-loaded DSC-MRI curve by using a conversion matrix. A, The reference region (green) on the EPI image of the unloaded DSC-MRI and pre-loaded DSC-MRI, respectively. B, The $\Delta R_{2}{ }^{*}$ curves of DSC-MRI are rearranged to $t$ (the number of time points) $\times n$ (the number of voxels in reference region) matrices. The conversion matrix is computed using SVD deconvolution. C, The non-deviated $\Delta R_{2}{ }^{*}$ curve of the unloaded DSC-MRI is estimated by multiplying the computed conversion matrix by the $\Delta R_{2}{ }^{*}$ curve of the pre-loaded DSC-MRI. D, The estimated $\Delta R_{2}{ }^{*}$ curve of the unloaded DSC-MRI and corresponding measured $\Delta R_{2}{ }^{*}$ curve of the unloaded DSC-MRI from the right hemisphere in a normal rat

where $\overline{\Delta R_{2}^{*}(t)}$ represents the averaged $\Delta R_{2}{ }^{*}$ curve for brain tissue regions with intact BBB, $\alpha$ is a time scaling factor, and $\tau$ is the arrival time. $\overline{\Delta R_{2}^{*}(t)}$ was computed by averaging the $\Delta R_{2}{ }^{*}$ curves from the contralateral hemisphere.

To estimate the proposed BBB leakage index from dual DSC-MRI, the following procedures were performed, as schematized in Figure 1. For the intact BBB region, the $\Delta R_{2}{ }^{*}$ curve is only affected by CA passage inside the vasculature embedded in the tissue of interest. Therefore, $\Delta R_{2}{ }^{*}$ curves from DSC-MRI can be described in the form of a discretized matrix as follows ${ }^{24}: \Delta R_{2}{ }^{*}=C_{A} \cdot r_{2}{ }^{*} \cdot C B F \cdot R$, where $r_{2}{ }^{*}$ is the transverse relaxivity of the CA, $R$ is the residue function, and $C_{A}$ is the matrix form of the arterial input function (AIF). Therefore, two sequential DSC-MRIs can be described as follows:

$$
\left\{\begin{array}{c}
\Delta R_{2,1 \mathrm{st}}^{*}=\mathrm{C}_{\mathrm{A}}^{1 \mathrm{st}} \cdot r_{2}^{*} \cdot \mathrm{CBF} \cdot R \\
\Delta R_{2,2 \mathrm{nd}}^{*}=\mathrm{C}_{\mathrm{A}}^{2 \mathrm{nd}} \cdot r_{2}^{*} \cdot \mathrm{CBF} \cdot R
\end{array}\right.
$$

where $\Delta R_{2,1 \text { st }}^{*}$ and $\Delta R_{2,2 \text { nd }}^{*}$ are the measured $\Delta R_{2}{ }^{*}$ curves for the first- (unloaded) and second-injected (pre-loaded) DSC-MRI acquisitions, respectively. $C_{A}^{1 \text { st }}$ and $C_{A}^{2 \text { nd }}$ are the AIFs for the corresponding DSC-MRIs, respectively.

In Equation 2, the residue function describes the fraction of injected CA remaining in the vasculature. Also, Equation 2 makes the assumption that the residue function is time invariant. ${ }^{25}$ Thus, if the same CA passes through the same tissue, the residue function is expected to be identical. Therefore, by assuming that the CBF and residue function $(R)$ do not significantly change for the unloaded and pre-loaded DSC-MRI acquisitions, the equations can be rearranged as follows:

$$
\Delta R_{2,1 \mathrm{st}}^{*}=C_{\mathrm{A}}^{1 \mathrm{st}} \cdot C_{\mathrm{A}}^{-1,2 \mathrm{nd}} \cdot \Delta R_{2,2 \mathrm{nd}}^{*}
$$

where $C_{A}^{-1,2 n d}$ is the inversion matrix of $C_{A}^{2 n d}$. In Equation $3, C_{A}^{1 s t} \cdot C_{A}^{-1,2 n d}$ describes the relationship between the unloaded and pre-loaded DSC-MRIs. Therefore, if the $C_{\mathrm{A}}^{1 \text { st }} \cdot C_{\mathrm{A}}^{-1,2 n d}$ matrix is known, $\Delta R_{2,2 n d}^{*}$ can be consistently converted to $\Delta R_{2,1 \text { st }}^{*}$. To calculate the conversion matrix $\left(C_{\mathrm{A}}^{1 \text { st }} \cdot C_{\mathrm{A}}^{-1,2 \text { nd }}\right), \Delta R_{2}{ }^{*}$ curves from the left hemisphere (contralateral hemisphere in the stroke group) can be considered an intact $\mathrm{BBB}$ region that satisfies Equation 3, as shown in Figure $1 \mathrm{~A}$ and $1 \mathrm{~B}$. 


$$
\left[\begin{array}{ccc}
\Delta R_{2,1 \mathrm{st}}^{*}(1,1) & \cdots & \Delta R_{2,1 \mathrm{st}}^{*}(1, n) \\
\vdots & \ddots & \vdots \\
\Delta R_{2,1 \mathrm{st}}^{*}(t, 1) & \cdots & \Delta R_{2,1 \mathrm{st}}^{*}(t, n)
\end{array}\right]=C_{\mathrm{A}}^{1 \mathrm{st}} \cdot C_{\mathrm{A}}^{-1,2 \mathrm{nd}}\left[\begin{array}{ccc}
\Delta R_{2,2 \mathrm{nd}}^{*}(1,1) & \cdots & \Delta R_{2,2 \mathrm{nd}}^{*}(1, n) \\
\vdots & \ddots & \vdots \\
\Delta R_{2,2 \mathrm{nd}}^{*}(t, 1) & \cdots & \Delta R_{2,2 \mathrm{nd}}^{*}(t, n)
\end{array}\right] .
$$

Next, the conversion matrix was calculated by multiplying the $\Delta R_{2}{ }^{*}$ data matrix from the unloaded DSC-MRI by the inversion matrix for the $\Delta R_{2}{ }^{*}$ data matrix from the pre-loaded DSC-MRI. The inversion matrix was computed based on singular value decomposition (SVD) with a threshold $\left(10^{-5}\right.$, this value was empirically set) of the maximum singular value. ${ }^{26}$ The estimation of $\Delta R_{2}{ }^{*}$ curves from the pre-loaded DSC-MRI was processed using a conversion matrix, as shown in Figure $1 \mathrm{C}$.

If CA is extravasated to the tissue, the estimated $\Delta R_{2}{ }^{*}$ curves will be different from the measured $\Delta R_{2}{ }^{*}$ curves. Therefore, the difference reflects the level of BBB damage and can be defined by the following equation:

$$
\text { leakage index }=\int_{0}^{t}\left(\text { estimated } \Delta R_{2}^{*}\left(t^{\prime}\right)-\text { measured } \Delta R_{2}^{*}\left(t^{\prime}\right)\right) / r_{2}^{*} d t^{\prime}
$$

After the derivation of proposed leakage index values following the above procedures, to compare the similarity between the $K_{2}$ map and leakage index map, the lesions with BBB leakage were defined as those that satisfied fixed criteria $\left(\Delta T_{1}>0, K_{2}>10^{-4}\right.$, and leakage index $\left.>0\right)$, and a scatter-plot between $K_{2}$ and leakage index values from the legion with damaged BBB was plotted. To further study the effects of the choice of intact BBB regions (reference region) on the $K_{2}$ or leakage index maps, four different ROls (contralateral hemisphere, contralateral cortex, contralateral striatum, and contralateral corpus callosum) were used in deriving $K_{2}$ and leakage index values. To offset any constant biases in the values of $K_{2}$ and leakage index within the contralateral hemisphere, all $K_{2}$ and leakage index values had with the averaged $K_{2}$ and leakage index values of the contralateral hemisphere subtracted.

To study the accuracy of the proposed method in estimating the extension of BBB leakage lesions, the correlation between the leakage map and two different $\Delta T_{1}$ maps ( $\Delta T_{1}$ map between pre-injection and 5 min after flushing the injection line $\left(\Delta T_{1,0 m i n}-5\right.$ min map), $\Delta T_{1}$ map between $5 \mathrm{~min}$ and $30 \mathrm{~min}$ after flushing the injection line ( $\Delta T_{1,5 \mathrm{~min}-30 \mathrm{~min}}$ map)) were mutually compared. Here, the $\Delta T_{1}$ map reflects the amount of $C A s$ passed through the disrupted BBB during a specific period. The hyperintense areas in the leakage index map were overlapped with the hyperintense areas in the $\Delta T_{1,0 \mathrm{~min}-5 \mathrm{~min}}$ or $\Delta T_{1,5 \mathrm{~min}-30 \mathrm{~min}}$ maps. To show the accuracy in estimating the degree of BBB leakage, the corresponding mean values of the leakage index and $\Delta T_{1,0 \mathrm{~min}-5 \mathrm{~min}}$ from the lesion with BBB damage were scatter-plotted. For the histological validation of the proposed method, the overlap between the leakage index map and Evans-blue-stained region was also directly compared.

To study the effects of the acquisition time of the leakage index map, five different time intervals (from $30 \mathrm{~s}$ to $165 \mathrm{~s}$ after CA injection) of $\Delta R_{2}{ }^{*}$ curves were used for the leakage index map. To further study the robustness of the estimated leakage index against the arrival time gap between the unloaded and pre-loaded DSC-MRI, leakage index maps with intentionally varied arrival times (time difference $=15 \mathrm{~s}$ ) were compared with matched ones. For the analysis of DCE-MRI acquisition, $K^{\text {trans }}$ values were evaluated using the extended Tofts model. ${ }^{27}$ The averaged DCE-MRI signal of the internal carotid artery (6 voxels) was considered as an AIF.

\section{3 | RESULTS}

To compare the sensitivities of DSC-based permeability $\left(K_{2}\right)$ mapping and DCE-based permeability $\left(K^{\text {trans }}\right)$ in characterizing subtly damaged BBB in ischemic lesions, Figure 2 shows the DCE-MRI and DSC-MRI results for a representative 1D reperfusion model. Hypointense areas on the ADC map and hyperintense areas on the $\Delta T_{1}$ map ( $T_{1}$ difference between pre-injection and 9 min after the CA injection for DCE-MRI or unloaded DSC-MRI) show the existence of edema and the location of BBB damage, respectively. There is a clear contrast between the contralateral hemisphere and the BBB damaged region on the $K_{2}$ map, but such contrast is not apparent on the corresponding $K^{\text {trans }}$ map. Figure $2 \mathrm{C}$ and $2 \mathrm{D}$ shows the averaged raw $\Delta R_{1}$ (from DCE) and $\Delta R_{2}{ }^{*}$ (from DSC) curve for the region with BBB damage, respectively. Signal enhancement due to CA extravasation $T_{1}$-shortening effects (black ellipses in Figures 2C and 2D) start to appear only after about $250 \mathrm{~s}$ (after injection) for DCE-MRI acquired $\Delta R_{1}$, but such enhancement was observed about $50 \mathrm{~s}$ after injection for DSC-MRI acquired $\Delta R_{2}{ }^{*}$ curves.

To show the efficacy of the proposed method for BBB-damaged ischemic brains, representative voxel-wise $\Delta R_{2}{ }^{*}$ curves of model-free (conversion matrix approach) leakage index estimation were shown for normal and ischemic brains on different reperfusion days. As a result of the dual DSC-MRI acquisitions, the estimated $\Delta R_{2}{ }^{*}$ curve from the pre-loaded DSC-MRI acquisition and the $\Delta R_{2}{ }^{*}$ curve from the unloaded DSC-MRI were compared in normal rats and tMCAO rats, as shown in Figure 3. Figure $3 \mathrm{~A}$ shows the quality of estimation for a single voxel $\Delta R_{2}{ }^{*}$ curve from a normal rat. Each $\Delta R_{2}{ }^{*}$ curve comes from a red arrowed voxel on the leakage index map (Figure 3A, middle). The estimated $\Delta R_{2}{ }^{*}$ curves from the left hemisphere (Figure 3A, left) and right hemisphere (Figure 3A, right) have high $r$-squared values (0.9730 and 0.9648 , respectively) for the normal brain with intact BBB. As shown in Figure 3B, there was no significant difference in $r$-squared values between the left and right hemispheres in the three normal rats $(p=0.109$, paired $t$-test with 95\% confidence level). However, as shown in Figure 3C and 3D, there were clear differences between measured (red dots, from the unloaded DSC-MRI) and estimated $\Delta R_{2}{ }^{*}$ curves (blue line, from the pre-loaded 
(A)

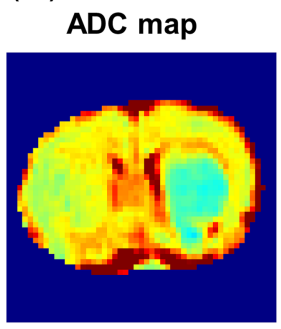

(B)

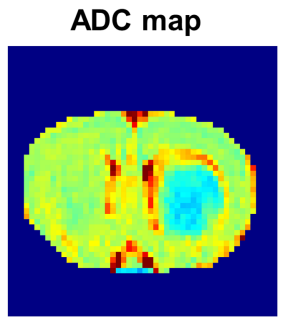

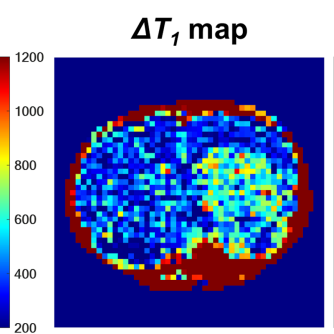

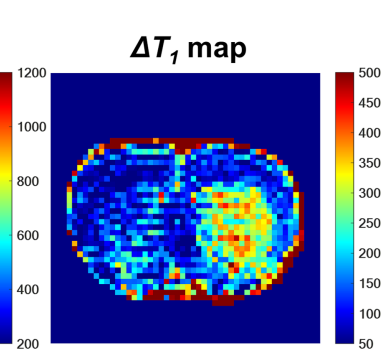

(C)
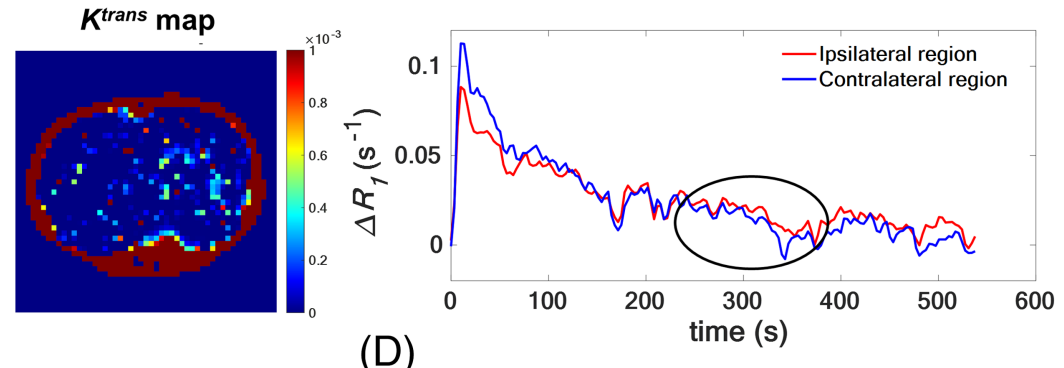

(D)
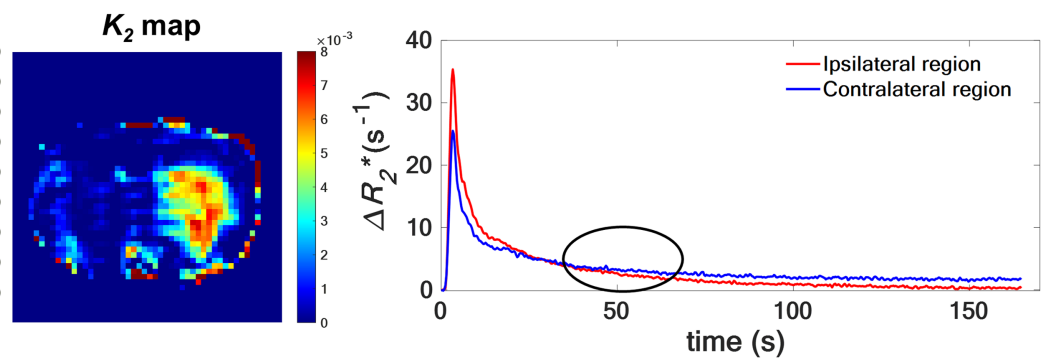

FIGURE 2 Comparison between DCE-MRI and DSC-MRI in $1 \mathrm{~h}$ MCAO $1 \mathrm{~d}$ reperfusion model. A, B, The ADC map, $\Delta T_{1}$ map, and corresponding permeability map, respectively. Both $\Delta T_{1}$ maps were acquired 9 min after CA injection and the total CA dose was the same $(0.3 \mathrm{mmol} / \mathrm{kg})$. C, D, The averaged relaxation rate curves ( 110 voxels were averaged) in ipsilateral and contralateral regions in DCE-MRI and DSC-MRI, respectively. The time at which the relaxation rate between the ipsilateral and contralateral regions begins to differ is indicated by a black ellipse, and is significantly shorter for $\Delta R_{2}{ }^{*}$ than $\Delta R_{1}$

(A)

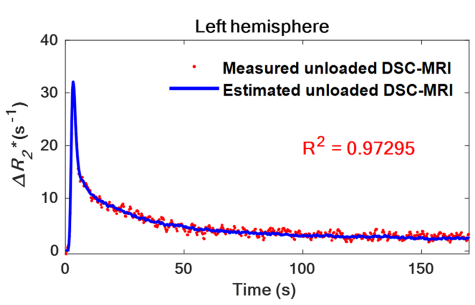

(C)

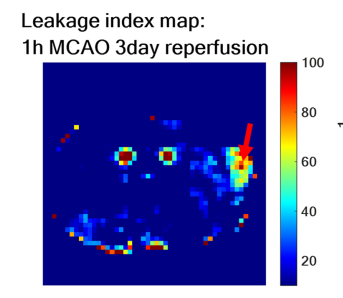

Leakage index map:

Normal rat
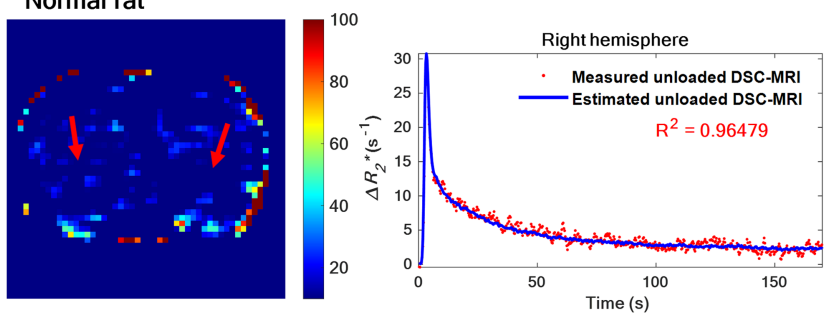

(D)

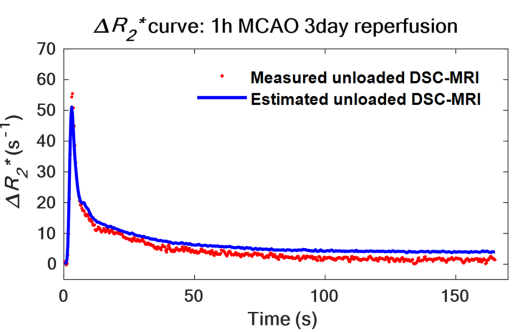

(B)

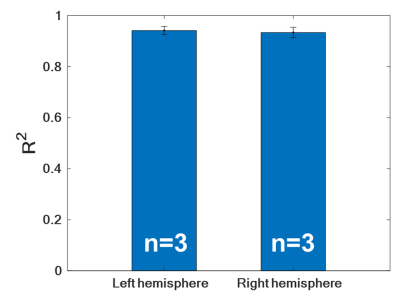

FIGURE 3 Representative voxel-wise $\Delta R_{2}{ }^{*}$ curves of model-free (conversion matrix approach) leakage index estimation for normal and ischemic brains. A, C, D, The leakage index map, estimated $\Delta R_{2}{ }^{*}$ curve for the unloaded DSC-MRI, and corresponding measured $\Delta R_{2}{ }^{*}$ curve of the unloaded DSC-MRI for normal, $3 \mathrm{~d}$, and $5 \mathrm{~d}$ reperfusion model rats, respectively. Each blue line and solid red dot show the estimated and measured $\Delta R_{2}{ }^{*}$ curve in a single voxel as indicated by the red arrow. B, Bar graphs of the $r$-squared value for the left and right hemispheres. The height and error bar represent the mean value and standard deviation of $r$-squared values for three normal rats. There is no significant difference between the left and right hemispheres $(p=0.109$, paired $t$-test)

DSC-MRI) for the $3 \mathrm{~d}$ and $5 \mathrm{~d}$ reperfusion models, due to BBB leakage in ischemic lesions. The degree of deviation appeared to be larger for the $5 \mathrm{~d}$ reperfusion model. Each representative $\Delta R_{2}{ }^{*}$ curve was obtained from one voxel indicated by the red arrow in each leakage index map (Figure 3C and 3D). 
(A) $T_{2}$-weighted image

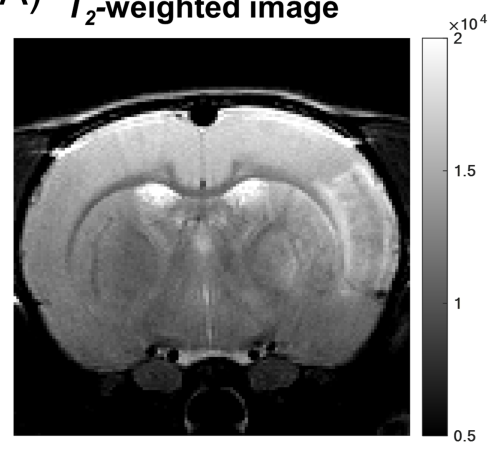

(C)

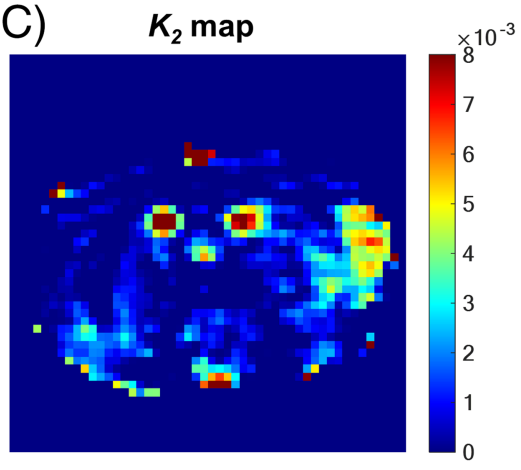

(B)

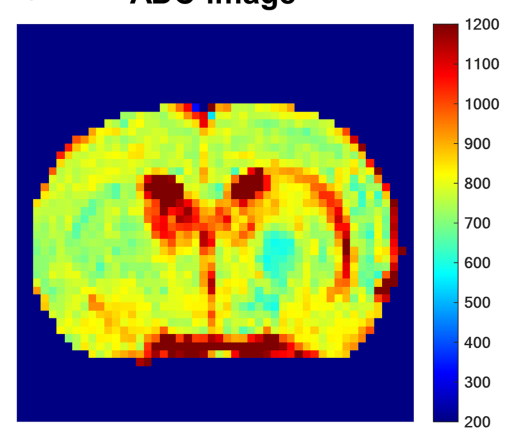

(D) Leakage index map

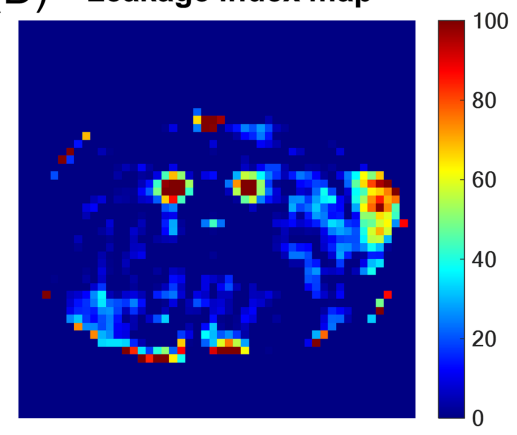

(E)

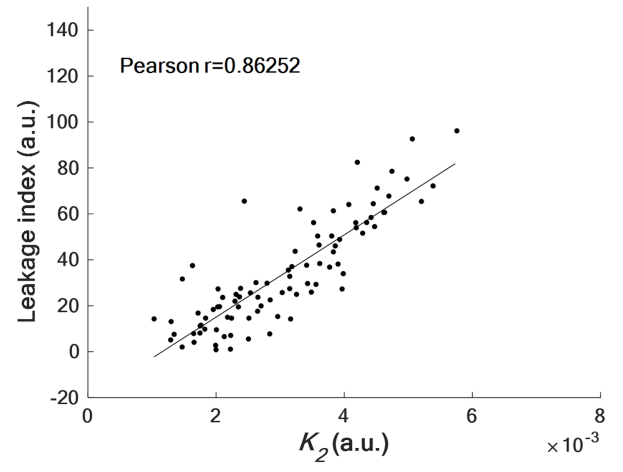

Pearson $r=0.649 \pm 0.158(n=16)$

FIG URE 4 Comparison between $K_{2}$ and leakage index maps. A-D, Representative $T_{2}$-weighted, ADC, $K_{2}$, and leakage index maps for a $3 d$ reperfusion model, respectively. E, Scatter-plot correlations between $K_{2}$ and leakage index values in a region with damaged BBB for the corresponding maps

\section{(A)}

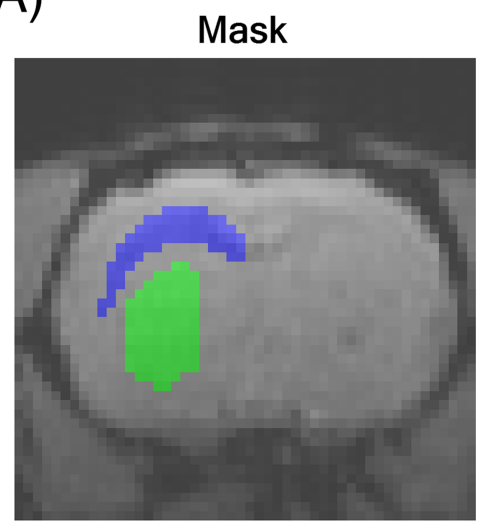

(B)
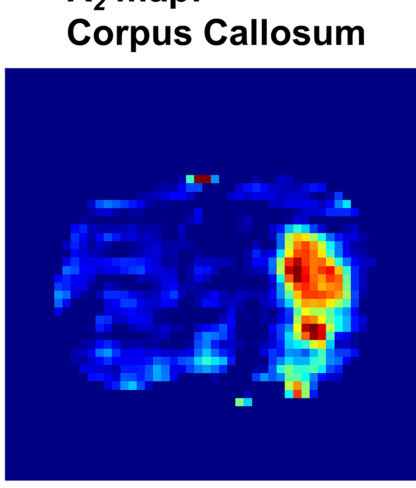

(D)
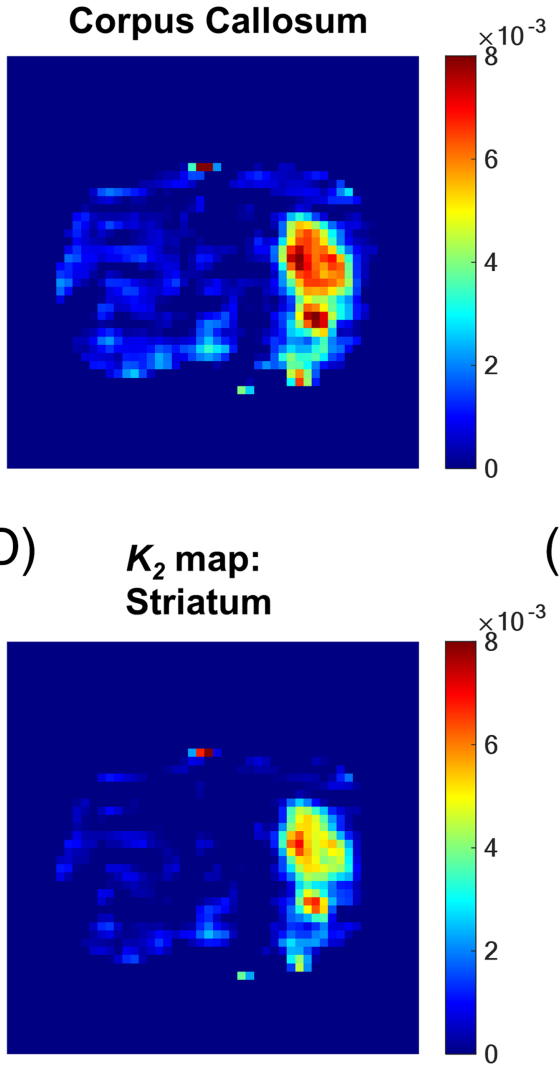

(C) Leakage index map: Corpus Callosum

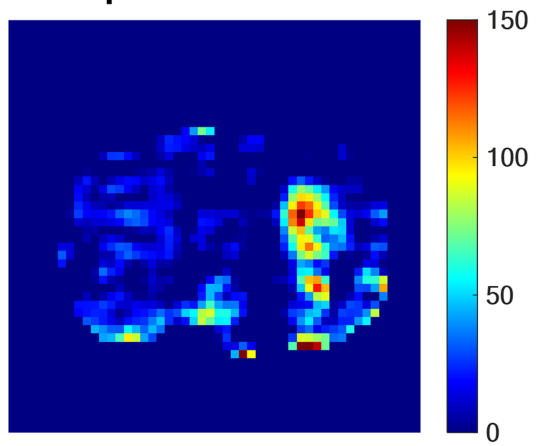

(E) Leakage index map: Striatum

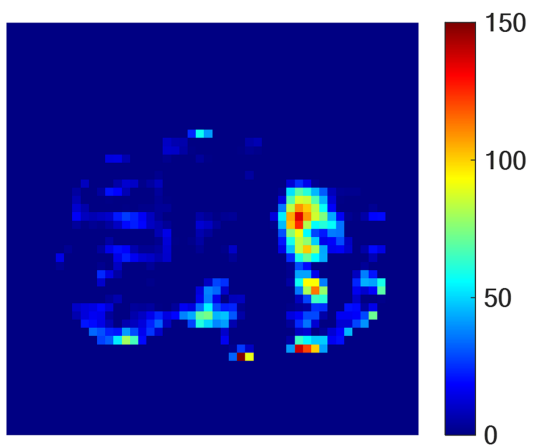

FIGURE 5 The representative $K_{2}$ and leakage index map with the choice of different reference regions. B-E, $K_{2}$ or leakage index maps with the corpus callosum or striatum as the reference region. A, The corresponding regions are marked in blue and green, respectively 
To address this similarity between the conventional $K_{2}$ map and the proposed leakage index map, Figure 4 shows the visual comparisons and associated correlation scatter-plot for a representative model. Figure $4 \mathrm{C}$ and $4 \mathrm{D}$ shows the $K_{2}$ and leakage index maps for the $3 \mathrm{~d}$ reperfusion model, respectively. The $T_{2}$-weighted image and ADC map of the corresponding slice are shown in Figure 4A and 4B, respectively. The $K_{2}$ and leakage index values in the regions with BBB damage show good mutual correlation (Pearson's $r=0.8625$ ), as shown in Figure 4E. The mean \pm standard deviation of Pearson's $r$ for 16 rats were $0.649 \pm 0.158$.

To demonstrate the repeatability of the proposed method against the different choices of reference ROI selection, Figure 5 shows the representative mapping results comparing the ROI dependences of the reference region for $K_{2}$ and the leakage index map. Figure $5 \mathrm{~B}$ and $5 \mathrm{C}$ shows $K_{2}$ and leakage index maps that were computed by considering the contralateral corpus callosum as a reference region (the reference region was used to calculate the conversion matrix and $\Delta R_{2}^{\bar{*}}(t)$ for the leakage index map and $K_{2}$ map, respectively.). The $K_{2}$ (Figure $5 \mathrm{D}$ ) and leakage index maps (Figure $5 \mathrm{E}$ ) were calculated using the contralateral striatum as the reference region. Reference regions for the corpus callosum and striatum in the contralateral brain are shown in blue and green in Figure 5A. A noticeable bias was observed for the $K_{2}$ map from the choice of different ROls of the reference region, while consistent leakage index maps were obtained regardless of the variation of reference ROIs.

To further validate the robustness of the leakage index map against reference region variations, Figure 6 shows the ROI dependence of the $K_{2}$ and leakage index map with all experimental ischemic brains on varying reperfusion days $(n=16)$. Selected reference regions for the cortex (red), corpus callosum (blue), striatum (green), and entire contralateral hemisphere (red, blue, green, and yellow) are shown in Figure 6A. The red area in Figure 6B corresponds to the representative ROI for the scatter-plots (Figure 6C and 6D). Figure 6C and 6D shows multiple scatter-plots between three different reference regions against the entire contralateral region for $K_{2}$ and leakage index in the region with BBB damage, respectively. The slopes of the scatter-plot for $K_{2}(0.94-1.07,14 \%$ error) were consistently lower than those for plots of the leakage index $(0.97-1.00,3 \%$ error), which shows the robustness of the leakage index estimated from dual DSC-MRI acquisitions.

Next, to verify the accuracy in estimating the extension and the degree of BBB-leakage by the proposed leakage index mapping, the overlap and correlation between the leakage index and $\Delta T_{1}$ maps are shown in Figure 7. Figure 7A shows the detailed MRI scan procedure
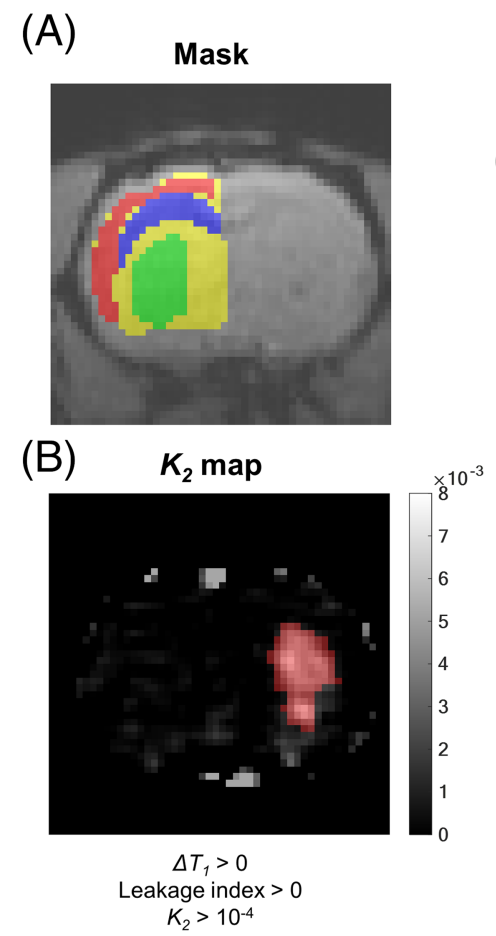

(C)

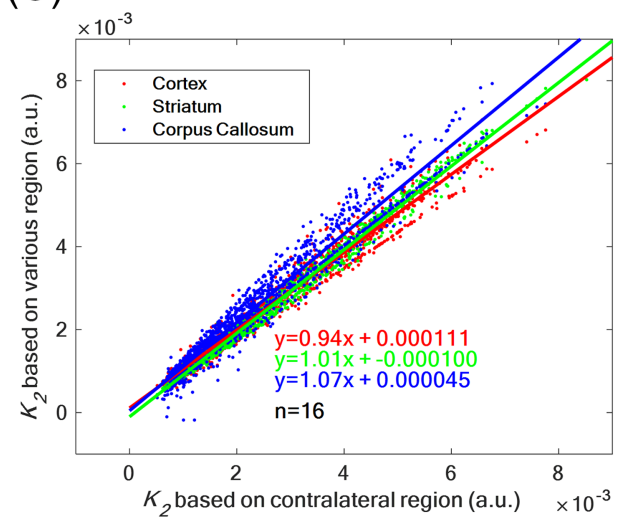

(D)

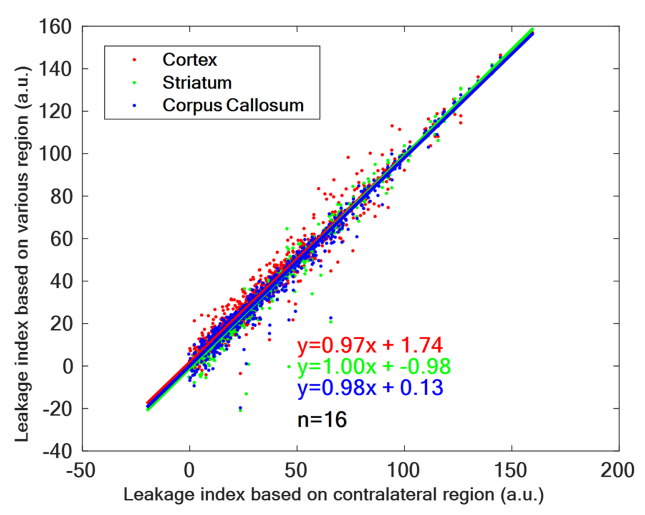

FIG URE 6 Variations of $K_{2}$ and leakage index values with various reference regions. C, D, Correlation scatter-plot for $K_{2}$ and leakage index values, respectively, between the values derived from various regions (cortex, striatum, and corpus callosum) with respect to the values derived from the contralateral region. The red, green, and blue colors in each scatter-plot indicate the $K_{2}$ or leakage index values chosen with the cortex, striatum, or corpus callosum as the reference region; the corresponding region is marked in the same color in A. A, The area in the contralateral hemisphere, which includes the red, green, blue, and yellow areas. Only the $K_{2}$ or leakage index values in the region with BBB damage are used for the scatter-plot; the region with BBB damage is defined based on the same criteria $\left(\Delta T_{1}>0, K_{2}>10^{-4}\right.$, leakage index $\left.>0\right)$. B, The corresponding region is shown in red 
(A)

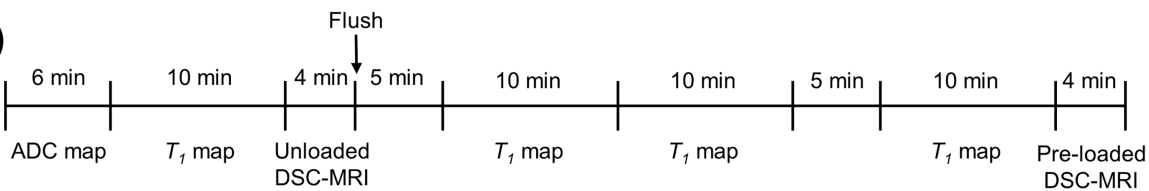

(B) Leakage index map

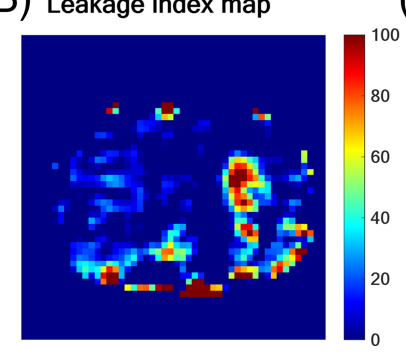

(C) $\Delta T_{1,5 \mathrm{~min}-30 \mathrm{~min}} \mathrm{map}$

(D) $\Delta T_{1, \text { omin-5min }}$ map

DSC-MRI
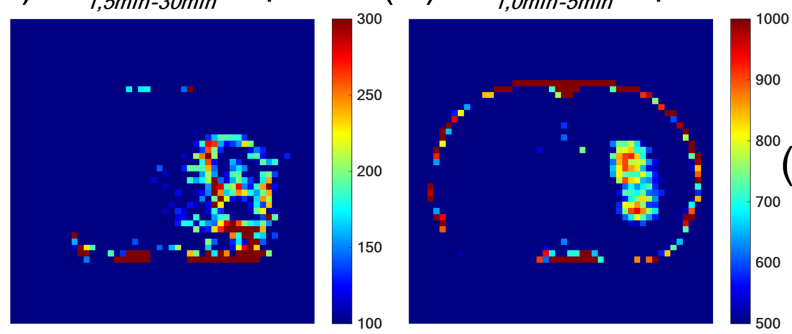

(E)

Combined mask Dice correlation $=0.1301$

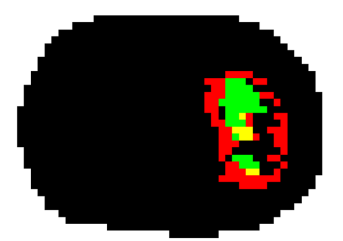

(F) Combined mask Dice correlation $=0.6897$

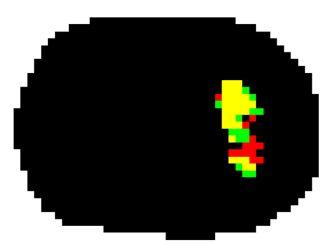

(G)

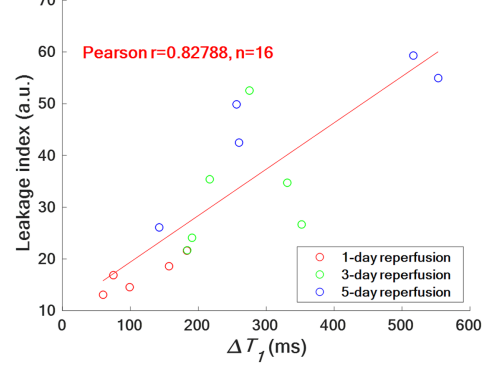

FIGURE 7 The correlation between $\Delta T_{1}$ and leakage index map for the extension and the level of BBB leakage. A, The MRI scan scheme for the $\mathrm{TMCAO}$ group. B-D, The representative leakage index map, $\Delta T_{1}$ between $5 \mathrm{~min}$ after and $30 \mathrm{~min}$ after flushing the injection line $\left(\Delta T_{1,5 \mathrm{~min}-30 \mathrm{~min}}\right)$, and $\Delta T_{1}$ between pre-injection and $5 \mathrm{~min}$ after the flush $\left(\Delta T_{1,0 \mathrm{~min}-5 \mathrm{~min}}\right)$ for a $5 \mathrm{~d}$ reperfusion rat model, respectively. $E$, $F$, The similarity of threshold masks between the leakage index map and $\Delta T_{1,5 \mathrm{~min}-30 \mathrm{~min}}$, or between the leakage index map and $\Delta T_{1,0 \mathrm{~min}-5 \mathrm{~min}}$, respectively. The red, green, and yellow-colored areas represent the threshold mask from the $\Delta T_{1}$ map $\left(\Delta T_{1,5 \mathrm{~min}-30 \mathrm{~min}}>180 \mathrm{~ms}\right.$ and $\left.\Delta T_{1,0 \mathrm{~min}-5 \mathrm{~min}}>725 \mathrm{~ms}\right)$, the leakage index map ( $>50$ a.u.), and the area where the two masks overlap, respectively. G, The correlation scatter-plot between the mean value of the leakage index and $\Delta T_{1,0 \mathrm{~min}-5 \mathrm{~min}}$ in the region with BBB damage in 16 rats. Two points (one point is $1 \mathrm{~d}$ reperfusion [183, 21.6] and the other is $3 \mathrm{~d}$ reperfusion $[184,21.6])$ overlap in $\mathrm{G}$

for the tMCAO group. Correspondingly, Figure 7B-D shows the leakage index map, $\Delta T_{1}$ map between 5 min and 30 min $\left(\Delta T_{1,5 \mathrm{~min}-30 \mathrm{~min}}\right.$ map), and $\Delta T_{1}$ map between pre-injection and $5 \mathrm{~min}\left(\Delta T_{1,0 \mathrm{~min}-5 \mathrm{~min}} \mathrm{map}\right)$ for $5 \mathrm{~d}$ reperfusion rats, respectively. Figure $7 \mathrm{E}$ and $7 \mathrm{~F}$ shows hyperintense regions of the leakage map (green area in $E$ and $F$ ), $\Delta T_{1,5 \mathrm{~min}-30 \mathrm{~min}}$ map (red area in $E$ ), and $\Delta T_{1,0 m i n-5 m i n}$ map (red area in $F$ ). The region of the overlap region between the leakage index and $\Delta T_{1}$ map is shown as a yellow area in Figure 7E and 7F. The hyperintense regions of the leakage index map were observed to be surrounded by the $\Delta T_{1,5 \min -30 \min }$ map, as shown in Figure $7 \mathrm{E}$ (Dice correlation $=0.1301$ ). However, the leakage index map and $\Delta T_{1,0 m i n-5 m i n}$ map show similar contrasts, as shown in Figure $7 F$ (Dice correlation $=0.6897$ ). Figure $7 G$ shows a significant correlation between the average leakage index and $\Delta T_{1,0 m i n}-5 \mathrm{~min}$ values for 16 rats (Pearson's $r=0.8279$ ).

Finally, for the histological validation of the proposed method, the overlap between the leakage index map and Evans blue-stained region is shown in Figure 8. Figure 8A-C shows the leakage index map, $T_{2}$-weighted image, and fluorescence image for the $1 \mathrm{~d}$ reperfusion model. The hyperintense regions of the leakage index map and fluorescence image correspond to the red and green areas in Figure 8D, respectively. The masks corresponded well, and the Dice correlation was 0.6263 . The observed overlaps and correlations of leakage index with respect to histology and $\Delta T_{1,0 \mathrm{~min}-5 \mathrm{~min}}$ map respectively validate the extension and the degree of BBB leakage, which are estimated from the dual DSC-MRI acquisition-derived leakage index.

Additionally, the effects of scan-time durations of the DSC-MRI on the leakage index map are shown in Supporting Information Figure S1. The extent of the leakage index map was observed to decrease with shorter scan-time duration. In particular, for the $105 \mathrm{~s}$ scan-time duration (Supporting Information Figure S1c), vascular permeability contrast at the bottom of the BBB damaged region was no longer detected. The effects of the arrival time gap between the unloaded and pre-loaded DSC-MRIs on the leakage index are shown in Supporting Information Figure S2. Supporting Information Figure S2a and S2c shows the quality of estimation of one voxel's $\Delta R_{2}{ }^{*}$ curves in normal tissue and ischemic lesions for $5 \mathrm{~d}$ reperfusion rats. The $\Delta R_{2}{ }^{*}$ curves in Supporting Information Figure S2a and S2c are from the voxels indicated by red arrows in Supporting Information Figure S2b. Supporting Information Figure S2d and S2f shows the quality of estimation of $\Delta R_{2}{ }^{*}$ curves, which have a $15 \mathrm{~s}$ arrival time difference between the unloaded and pre-loaded DSC-MRIs and show the same results in Supporting Information Figure S2a and S2c. Supporting 


\section{(A) Leakage index map}

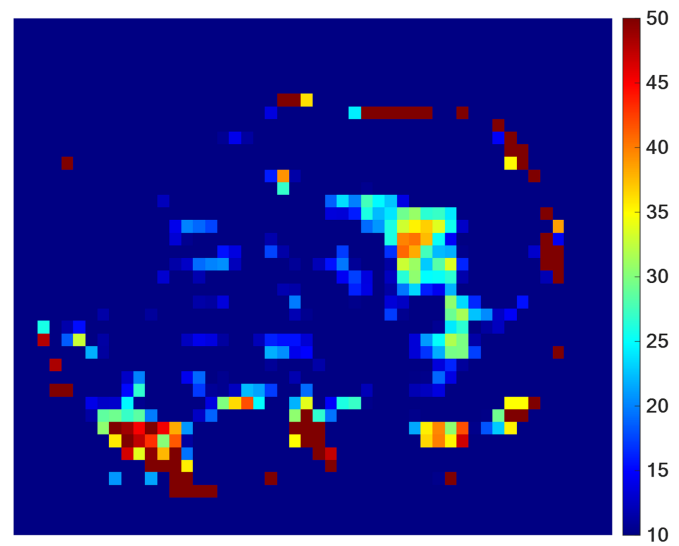

(C)

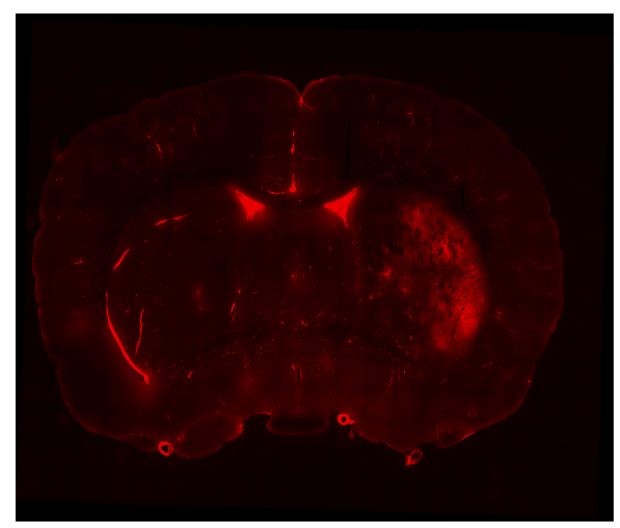

(B)

\section{$T_{2}$-weighted image}

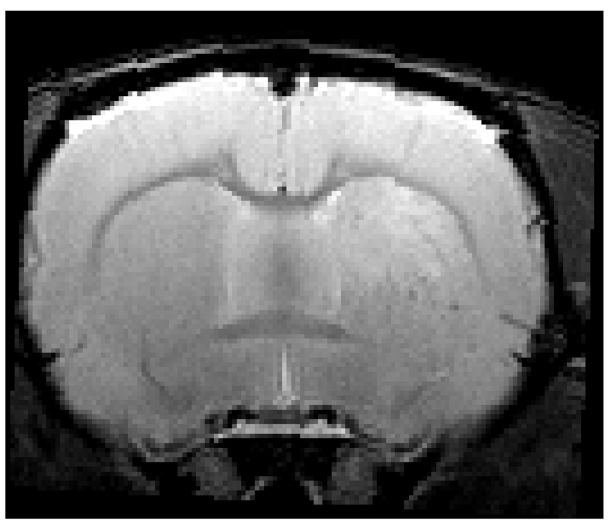

(D)
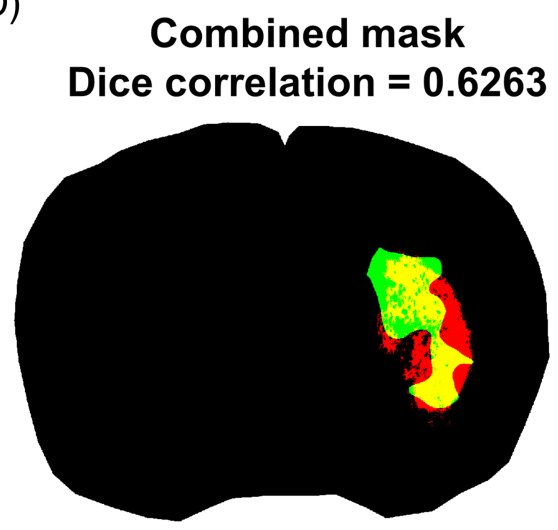

FIGURE 8 Comparison between the leakage index map and Evans-blue-stained image. A-C, Leakage index map, $T_{2}$-weighted image, and fluorescence image for the $3 \mathrm{~d}$ reperfusion model. D, Similarity between the leakage index map and Evans-blue-stained image. The red, green, and yellow areas represent the threshold mask from the Evans-blue-stained image, the leakage index map, and the area where the two masks overlap, respectively

Information Figure S2b and S2e shows similar vascular permeability contrast regardless of the arbitrarily imposed potential arrival time variabilities between the two DSC-MRIs.

\section{4 | DISCUSSION}

Although DCE-MRI is widely used for evaluating vascular permeability, previous studies have shown that DCE-MRI has limited sensitivity for evaluating subtle ischemic vascular permeability. ${ }^{11,28-30}$ A long DCE-MRI acquisition time ( 20 min) would enhance the contrast as a result of the increased amount of extravasated $C A .{ }^{31-33}$ However, extravascular extracellular space volume $\left(v_{\mathrm{e}}\right)$ and CA diffusion begin to affect the amount of extravasated CA over time and may not directly represent spatial vascular permeability. ${ }^{34}$ Therefore, to evaluate subtle vascular permeability, it will be more appropriate to minimize the duration of dynamic acquisition, while achieving a sufficiently large MR contrast to distinguish the BBB leakage lesion due to direct CA extravasations. In this study, the $\Delta R_{2}{ }^{*}$ curve based on DSC-MRI showed significant signal deviation due to CA leakage with a minimized duration ( $100 \mathrm{~s}$ ) of dynamic acquisition, which was apparently much shorter than the required duration for corresponding DCE-MRI acquisition. This is also probably because the longer $T_{R}$ and the larger $T_{2}{ }^{*}$ effects of DSC-MRI than DCE-MRI produce a higher signal-to-noise ratio (SNR) and contrast-to-noise ratio. Apart from the benefit for the SNR, decreasing the susceptibility difference between the tissue and vasculature with extravasating CA may further reduce $\Delta R_{2}{ }^{*}$ values of the unloaded DSC-MRI, in addition to $\Delta R_{1}$ effects.

Several studies have consistently shown that pre-loaded DSC-MRI acquisition provides a $\Delta R_{2}{ }^{*}$ curve with minimized CA extravasations. By using the conversion matrix obtained by comparing $\Delta R_{2}{ }^{*}$ curves between sequential DSC-MRI acquisitions of regions with intact BBB, the non-extravasated $\Delta R_{2}{ }^{*}$ curve of a single voxel can be extracted from the pre-loaded DSC-MRI for lesions with BBB leakage. As each voxel's $\Delta R_{2}{ }^{*}$ curves of unloaded and pre-loaded DSC-MRI originate from the same vascular structural information, the associated leakage index should be 
independent of blood volume, dispersion, or arrival time. Therefore, leakage index values appear to avoid the modulation problem faced by conventional $K_{2}$ model fits without the need to introduce a time scaling factor or time offset. In addition, the leakage index relies only on the relationship between the unloaded and pre-loaded DSC-MRI, with no need for pharmacokinetic modeling. Therefore, the leakage index may also be less error-prone due to discrepancies between the pharmacokinetic model and measured data.

The limitations of this study are as follows. First, the change in physiological state between the unloaded and pre-loaded DSC-MRI may alter $\mathrm{CBF}$ in Equation, which would potentially cause an error in the conversion matrix estimation. If changes occur throughout the brain, the effects on the conversion matrix are not significant. However, if there is a local CBF change in the lesion after the unloaded DSC-MRI, the conversion matrix could be incorrectly computed. Such a potential error can be further reduced by reducing the time interval between the unloaded and preloaded DSC-MRIs. By shortening the time interval between the unloaded and pre-loaded DSC-MRIs as much as possible, the local CBF changes between the unloaded and pre-loaded DSC-MRIs may be further minimized and a more accurate conversion matrix can be provided. Second, the effects of the remaining CA in blood on the leakage index have not been explored. To increase the accuracy of the conversion matrix, the interval between the unloaded and pre-loaded DSC-MRIs should be reduced as much as possible. However, if there is not sufficient time between the unloaded and pre-loaded DSC-MRI, the remaining CA from the unloaded DSC-MRI may shift the baseline DSC-MRI curves of the pre-loaded DSC-MRI. Therefore, to determine the optimal time interval, the effects of remaining CA in blood should be systematically studied. To achieve this, the error of leakage index may be evaluated by shortening the time interval between the unloaded and pre-loaded DSC-MRIs. If the effects of the time interval on the accuracy of leakage index are studied, the dual DSC-MRI method can be readily applied to patients because the concept of pre-loaded DSC-MRI is already well established in the clinic. Third, from a practical point of view, the dose of CA is limited. However, half the dose $(0.05 \mathrm{mmol} / \mathrm{kg})$ used in typical clinical DSC-MRI scans can be used for each injection in clinical dual DSC-MRIs studies. When calculating the conversion matrix, the closer the dual DSC-MRI conditions, the more accurate the conversion matrix would be. However, considering the linear response of $\Delta R_{2}{ }^{*}$ with different doses, ${ }^{27}$ the contrast may be improved by optimizing injection doses between dual DSC-MRI acquisitions.

In summary, the feasibility of estimating a non-extravasated $\Delta R_{2}{ }^{*}$ curve in a lesion with BBB leakage based on the pre-loaded DSC-MRI was demonstrated. The corresponding leakage index was computed by evaluating the difference between the non-extravasated (estimated from the pre-loaded DSC-MRI) and extravasated (measured from the unloaded DSC-MRI) $\Delta R_{2}{ }^{*}$ curves. Such leakage index maps showed similar contrasts to conventional $K_{2}$ maps but were observed to be more robust against the choice of reference region with intact BBB. The validity of the leakage index as a measure of the degree of inter-subject BBB integrity was validated with corresponding in vivo $\Delta T_{1}$ values. The area of the elevated leakage index matched well with the corresponding histological Evans-blue-stained image. While dual CA injections are frequently performed for pre-loaded DSC-MRI in preclinical and clinical settings, the proposed model-free leakage index estimation technique from dual DSC-MRI may improve the robust quantification of inter-subject vascular permeability, especially for lesions with subtle BBB damage in ischemic brains.

\section{FUNDING INFORMATION}

This work was partially supported by National Research Foundation of Korea grants from the Korean government (2018R1A6A1A03025810, 2018M3C7A1056887). This study was partially supported by a grant of the Korea Health Industry Development Institute (HI14C1135). This research was also partially supported by the '2021 Joint Research Project of Institutes of Science and Technology'.

\section{REFERENCES}

1. Khatri R, McKinney AM, Swenson B, Janardhan V. Blood-brain barrier, reperfusion injury, and hemorrhagic transformation in acute ischemic stroke. Neurology. 2012;79(13 Supplement 1):S52-S57.

2. Warach S, Latour LL. Evidence of reperfusion injury, exacerbated by thrombolytic therapy, in human focal brain ischemia using a novel imaging marker of early blood-brain barrier disruption. Stroke. 2004;35(11 Suppl 1):2659-2661.

3. Brouns R, De Deyn PP. The complexity of neurobiological processes in acute ischemic stroke. Clin Neurol Neurosurg. 2009;111(6):483-495.

4. Neumann-Haefelin C, Brinker G, Uhlenküken U, Pillekamp F, Hossmann K-A, Hoehn M. Prediction of hemorrhagic transformation after thrombolytic therapy of clot embolism: an MRI investigation in rat brain. Stroke. 2002;33(5):1392-1398.

5. Jickling GC, Liu D, Stamova B, et al. Hemorrhagic transformation after ischemic stroke in animals and humans. J Cereb Blood Flow Metab. 2014;34(2): 185-199.

6. Zhang N, Zhang L, Qiu B, Meng L, Wang X, Hou BL. Correlation of volume transfer coefficient $\mathrm{K}^{\text {trans }}$ with histopathologic grades of gliomas. J Magn Reson Imaging. 2012;36(2):355-363.

7. Haselhorst R, Kappos L, Bilecen D, et al. Dynamic susceptibility contrast MR imaging of plaque development in multiple sclerosis: application of an extended blood-brain barrier leakage correction. J Magn Reson Imaging. 2000;11(5):495-505.

8. Boxerman JL, Schmainda KM, Weisskoff RM. Relative cerebral blood volume maps corrected for contrast agent extravasation significantly correlate with glioma tumor grade, whereas uncorrected maps do not. Am J Neuroradiol. 2006;27(4):859-867.

9. Tofts PS, Brix G, Buckley DL, et al. Estimating kinetic parameters from dynamic contrast-enhanced $\mathrm{T}_{1}$-weighted MRI of a diffusable tracer: standardized quantities and symbols. J Magn Reson Imaging. 1999;10(3):223-232.

10. Essig M, Shiroishi MS, Nguyen TB, et al. Perfusion MRI: the five most frequently asked technical questions. Am J Roentgenol. 2013;200(1):24-34.

11. Jin S, Han S, Stoyanova R, Ackerstaff E, Cho H. Pattern recognition analysis of dynamic susceptibility contrast (DSC)-MRI curves automatically segments tissue areas with intact blood-brain barrier in a rat stroke model: a feasibility and comparison study. J Magn Reson Imaging. 2020;51(5): 1369-1381. 
12. Kiselev VG. Transverse relaxation effect of MRI contrast agents: a crucial issue for quantitative measurements of cerebral perfusion. J Magn Reson Imaging. 2005;22(6):693-696.

13. Calamante F, Thomas DL, Pell GS, Wiersma J, Turner R. Measuring cerebral blood flow using magnetic resonance imaging techniques. J Cereb Blood Flow Metab. 1999;19(7):701-735.

14. Østergaard L. Principles of cerebral perfusion imaging by bolus tracking. J Magn Reson Imaging. 2005;22(6):710-717.

15. Calamante F, Gadian DG, Connelly A. Quantification of perfusion using bolus tracking magnetic resonance imaging in stroke: assumptions, limitations, and potential implications for clinical use. Stroke. 2002;33(4):1146-1151.

16. Haselhorst R, Kappos L, Bilecen D, et al. Dynamic susceptibility contrast MR imaging of plaque development in multiple sclerosis: application of an extended blood-brain barrier leakage correction. J Magn Reson Imaging. 2000;11(5):495-505.

17. Leigh R, Jen SS, Varma DD, Hillis AE, Barker PB. Arrival time correction for dynamic susceptibility contrast MR permeability imaging in stroke patients. PLoS ONE. 2012;7(12):e52656. https://doi.org/10.1371/journal.pone.0052656

18. Boxerman JL, Prah DE, Paulson ES, Machan JT, Bedekar D, Schmainda KM. The role of preload and leakage correction in gadolinium-based cerebral blood volume estimation determined by comparison with MION as a criterion standard. Am J Neuroradiol. 2012;33(6):1081-1087.

19. Jin S, Kang M, Cho H. Cerebral blood perfusion deficits using dynamic susceptibility contrast MRI with gadolinium chelates in rats with post-ischemic reperfusion without significant dynamic contrast-enhanced MRI-derived vessel permeabilities: a cautionary note. PloS ONE. 2018;13(7):e0201076. https://doi.org/10.1371/journal.pone.0201076

20. Do'sa E, Guillaume DJ, Haluska M, et al. Magnetic resonance imaging of intracranial tumors: intra-patient comparison of gadoteridol and ferumoxytol. Neuro Oncol. 2011;13(2):251-260.

21. Schmainda KM, Rand SD, Joseph AM, et al. Characterization of a first-pass gradient-echo spin-echo method to predict brain tumor grade and angiogenesis. Am J Neuroradiol. 2004;25(9):1524-1532.

22. Wang HL, Lai TW. Optimization of Evans blue quantitation in limited rat tissue samples. Sci Rep. 2014;4:1-7.

23. Meng X, Fisher M, Shen Q, Sotak CH, Duong TQ. Characterizing the diffusion/perfusion mismatch in experimental focal cerebral ischemia. Ann Neurol. 2004;55(2):207-212.

24. Ostergaard L, Weisskoff RM, Chesler DA, Gyldensted C, Rosen BR. High resolution measurement of cerebral blood flow using intravascular tracer bolus passages. Part I: Mathematical approach and statistical analysis. Magn Reson Med. 1996;36(5):715-725.

25. Kosior JC, Frayne R. Perfusion parameters derived from bolus-tracking perfusion imaging are immune to tracer recirculation. $J$ Magn Reson Imaging. 2010;31(3):753-756.

26. Wirestam R, Andersson L, Østergaard L, et al. Assessment of regional cerebral blood flow by dynamic susceptibility contrast MRI using different deconvolution techniques. Magn Reson Med. 2000;43(5):691-700.

27. Tofts PS. Modeling tracer kinetics in dynamic Gd-DTPA MR imaging. J Magn Reson Imaging. 1997;7(1):91-101.

28. Lin C, Chang C, Cheung W, et al. Dynamic changes in vascular permeability, cerebral blood volume, vascular density, and size after transient focal cerebral ischemia in rats: evaluation with contrast-enhanced magnetic resonance imaging. J Cereb Blood Flow Metab. 2008;28(8):1491-1501.

29. Armitage PA, Farrall AJ, Carpenter TK, Doubal FN, Wardlaw JM. Use of dynamic contrast-enhanced MRI to measure subtle blood-brain barrier abnormalities. Magn Reson Imaging. 2011;29(3):305-314.

30. Montagne A, Toga AW, Zlokovic BV. Blood-brain barrier permeability and gadolinium: benefits and potential pitfalls in research. JAMA Neurol. 2016; 73(1):13-14.

31. Wardlaw JM, Farrall A, Armitage PA, et al. Changes in background blood-brain barrier integrity between lacunar and cortical ischemic stroke subtypes. Stroke. 2008;39(4):1327-1332.

32. Thrippleton MJ, Backes WH, Sourbron S, et al. Quantifying blood-brain barrier leakage in small vessel disease: review and consensus recommendations. Alzheimers Dement. 2019;15(6):840-858.

33. Cramer SP, Larsson HBW. Accurate determination of blood-brain barrier permeability using dynamic contrast-enhanced T1-weighted MRI: a simulation and in vivo study on healthy subjects and multiple sclerosis patients. J Cereb Blood Flow Metab. 2014;34(10):1655-1665.

34. Cuenod CA, Balvay D. Perfusion and vascular permeability: basic concepts and measurement in DCE-CT and DCE-MRI. Diagn Interv Imaging. 2013; 94(12):1187-1204.

\section{SUPPORTING INFORMATION}

Additional supporting information may be found online in the Supporting Information section at the end of this article.

How to cite this article: Jin S, Cho HJ. Model-free leakage index estimation of the blood-brain barrier using dual dynamic susceptibility contrast MRI acquisition. NMR in Biomedicine. 2021;34(10):e4570. https://doi.org/10.1002/nbm.4570 